\title{
YOUNG INVESTIGATOR AWARDEES
}

NINTH WORKSHOP ON VITAMIN D

\section{Dilon Daniel}

Department of Chemistry

University of California-Riverside

Riverside, California, USA

\section{Helene Defacque}

Department of Cellular Biology

Universite Montpellier II

Montpellier Cedex, France

\section{F. Jeffrey Dilworth}

Department of Biochemistry

Queen's University

Kingston, Ontario, Canada

\section{AnneMarie Gagnon \\ Department of Biochemistry \\ University of Ottawa \\ Ottawa, Ontario, Canada}

\section{Mireille Lambert}

Hopital Robert Debre

Paris, France

\section{Gail Marchetto}

Department of Biochemistry

University of California-Riverside

Riverside, California, USA

\section{Chantal Mathieu}

Lab. Exper. Med. \& Endocrinology Katholieke Universiteit Leuven

Leuven, Belgium

\section{Theresa Matkovits}

Dept of Biochemistry \& Molecular Biology

New Jersey Medical School

Newark, New Jersey, USA

\section{Hans Postlind}

Department of Pharmaceutical Biosciences University of Uppsala

Uppsala, Sweden

\section{Trudy Vink-van Wijngaarden \\ Department of Internal Medicine III \\ Erasmus University \\ Rotterdam, The Netherlands}

\section{Susana B. Zanello}

Departamento de Biologia

Universidad Nacional del Sur

Bahia Blanca, Argentina

\section{Hong Zhao}

Department of Chemistry

New York University

New York, New York, USA 